\title{
Demographic, practice and clinical management characteristics of osteopaths referring to podiatrists: secondary analysis of a nationally representative sample of Australian osteopaths
}

Michael Fleischmann ${ }^{1,2^{*}}$, Brett Vaughan ${ }^{2,3,4}$, Adam Bird $^{5}$, Sandra Grace ${ }^{4}$, Kylie Fitzgerald $^{3}$ and Gopi McLeod ${ }^{2,4}$

\begin{abstract}
Background: Interprofessional care is paramount in contemporary healthcare practice. How different professions interact, and the characteristics of those practitioners who practice in an interprofessional way are rarely described in the literature. The aim of the current work was to identify the demographic, practice and clinical management characteristics of Australian osteopaths who report referring to podiatrists.

Methods: The study was a secondary analysis of data from the Osteopathy Research and Innovation Network (ORION). Inferential statistics were generated to identify statistically significant demographic, practice and clinical management characteristics associated with referrals to podiatrists by Australian osteopaths.

Results: Nine-hundred and ninety-two Australian osteopaths responded to the questionnaire. Sending referrals to a podiatrist was reported by 651 participants (65.6\%). Female Australian osteopaths were less likely to report referring to podiatrists compared to male osteopaths $(O R 0.76,95 \% \mathrm{Cl} 0.59-0.99)$. Australian osteopaths who reported referring to podiatrists were more likely to report receiving referrals from podiatrists $(O R$ 9.75, 95\% Cl 6.98-13.61), use orthopaedic testing in patient assessment $(O R 7.62,95 \% \mathrm{Cl}$ 2.82-20.60), and often treat patients with postural disorders (OR 1.71, $95 \% \mathrm{Cl} 1.03-2.26)$, compared to osteopaths who do not refer to podiatrists.

Conclusion: This study provides initial evidence for the referral relationship between Australian osteopaths and podiatrists. Further work could explore the nature of these referrals, including the complaints resulting in referral and outcomes of care. This information will be useful to those involved in health policy development and the professions advocating for their role in the wider healthcare system.
\end{abstract}

\section{Background}

Multidisciplinary care has been associated with benefits for patients and health professionals alike. These benefits include improved health outcomes, patient satisfaction,

*Correspondence: michael.flesichmann@vu.edu.au

${ }^{2}$ School of Public Health, University of Technology Sydney, Sydney,

Australia

Full list of author information is available at the end of the article efficient use of resources, and job satisfaction for team members [1]. Working in a multidisciplinary care environment can be significantly influenced by context [2], particularly practice location and co-location with other health professionals $[3,4]$. Health professionals located in tertiary care environments can readily engage in multidisciplinary care, given the ease of access to other health professionals. In the primary care environment, however, 
this immediacy of access may be more challenging to achieve and may require different pathways for patients to involve other health professionals in their care.

Australian podiatrists work in both public and private health contexts where they provide care for a range of lower limb conditions. In contrast, Australian osteopaths are predominantly located in private health care settings, where they provide care for a range of musculoskeletal complaints, including those affecting the lower limb [5-7]. Both professions are government registered in Australia, with accredited pre-registration programmes $[8,9]$. At the end of 2019 , there were 5509 registered podiatrists, with nearly $60 \%$ identifying as female [10], and 2723 registered osteopaths, with $55 \%$ identifying as female [11]. At the time over half of each the practitioners in each profession were less than 40 years of age. It is not possible to ascertain the practice location (public versus private) from this registration data; however, other works suggest that in Australia 90\% of osteopaths [5] and approximately $72 \%$ of podiatrists [12] are located in private practice.

Australian podiatrists and osteopaths share several practice commonalities - in particular, the care of lower limb musculoskeletal complaints [5-7]. In Australia, services provided by both professions are included in the Medicare Chronic Disease Management (CDM) plan scheme [13], whereby patients can access, under Medicare, up to five consultations with allied health professionals(e.g. podiatrist, dietician, physiotherapist) per year to assist with the management of a chronic complaint, including musculoskeletal issues. This scheme entitles patients to a rebate from the Australian Government to assist with the costs of their care [13]. The CDM scheme presents an opportunity for osteopaths and podiatrists to work as part of a patient's multidisciplinary care team. Menz [14] reported that in the 2004-2008 period over 1.3 million consultations for podiatry care and 82,486 consultations with osteopaths were funded over the same period through the CDM scheme [15], with the rebates facilitated through the Medicare EasyClaim system. The almost ten-fold difference between the number of podiatry consultations and the number of osteopathy consultations through the CDM scheme is likely to be due to podiatrists having a narrower specialisation (foot care), and hence less competition compared with osteopaths, who are just one type of provider of musculoskeletal care under the CDM scheme.

Practice-based research networks (PBRNs) foster research, develop practice-relevant research questions, and assist knowledge translation to improve clinical care $[16,17]$. PBRNs have been used both in Australia and internationally across the medical and allied health professions [5, 16, 18-21]. The current study explored the demographic, practice and clinical-management characteristics of the practice of Australian osteopaths who send referrals to, and receive referrals from, podiatrists through the Osteopathy Research and Innovation Network (ORION) - the largest voluntary nationally representative PBRN in osteopathy worldwide [5, 22]. Little is known about the patterns of patient referrals between the osteopathy and podiatry professions (amongst other health professions) in Australia. An emerging picture of referrals for both of these professions in Australia suggests that referrals are made to and from a range of health professionals $[5,6,23]$. However, the characteristics of Australian osteopaths who send referrals to other health professionals has not been explored to date. The data from this secondary analysis of the ORION PBRN will not only contribute to our understanding of how these two professions work together in the Australian healthcare system but also inform interprofessional education in pre- and post-registration training programmes and assist with the development of health policy for interprofessional care.

\section{Method \\ Sample}

Nine-hundred and ninety-two $(N=992)$ responses were received, representing $49 \%$ of the osteopathy profession at time of completion in July to December 2016. Participants were required to be registered osteopaths practising in Australia. Study participants were recruited through the Australian osteopathy professional association (Osteopathy Australia) and by word of mouth. The baseline sample of participants (including that the sample is nationally representative on a number of key indicators) is extensively described elsewhere [5].

\section{Questionnaire}

A questionnaire was developed to establish ORION baseline data and this data is described elsewhere [5, 22]. Participants were invited to complete a self-report 27-item questionnaire designed to collect a range of demographic (e.g., age, gender, highest osteopathy degree, other degrees, length of time in clinical practice), clinical (e.g., presenting conditions, patient groups treated, techniques and adjuncts applied) and practice characteristics (e.g., patient care hours per week, visits per week, co-located with other health professionals, referral to and/or from other health professionals). The questionnaire did not explore other individual practitioner characteristics such as ethnicity or income level. The analysis presented in this paper focuses on the demographic, practice and clinical management characteristics of osteopaths associated with referrals to Australian podiatrists. 


\section{Statistical analyses}

Participant responses were analysed based on whether the respondent reported sending patient referrals to podiatrists (outcome variable). Other variables were analysed in relation the independent variables, using independent $\mathrm{t}$-tests for continuous variables and chi-square tests for categorical variables. Effect size and unadjusted odds ratios were calculated for statistically significant variables. Alpha was set at 0.05 . All descriptive statistical analyses, t-tests, and chi-square tests were performed using SPSS version 25 (IBM Corp., Armonk, NY, USA).

\section{Results}

Responses were received from all 992 osteopaths who responded to the ORION questionnaire question regarding sending referrals to a podiatrist (yes/no), with no missing data. One hundred and forty-seven respondents $(n=147,14.8 \%)$ reported being co-located with a podiatrist. Sending referrals to a podiatrist was reported by 651 participants $(65.6 \%)$ and receiving referrals from a podiatrist by 471 participants (47.5\%). The demographic characteristics of Australian osteopaths who reported sending referrals are provided in Table 1. Female osteopaths were significantly less likely than male osteopaths to send referrals to podiatrists (OR $0.76,95 \% \mathrm{CI} 0.59-0.99)$, and those osteopaths who reported referring patients to podiatrists were likely to be younger with a small effect size $(p<0.01, \mathrm{~d}=0.17)$.

Osteopaths who reported being co-located with a podiatrist, were more likely to report sending referrals to a podiatrist (OR $3.08,95 \% \mathrm{CI} 1.94-4.87$ ) or exercise physiologist (OR 2.27, 95\%CI 1.43-3.59), compared to colleagues who did not report referring to podiatrists (Table 2). Australian osteopaths who reported sending referrals to podiatrists were also over four times more likely to send referrals to general practitioners $(O R$ 4.62, 95\%CI 3.05-7.01) and more than twice as likely to send referrals to a range of medical, allied health and complementary medicine professionals (Table 2). Osteopaths who reported sending referrals to podiatrists over nine times more likely to receive report receiving referrals from a podiatrist, compared to osteopaths who did not report referring (OR 9.75, 95\%CI 6.98-13.61). Orthopaedic testing was more likely to be used by osteopaths who

Table 1 Comparison of practitioner characteristics of Australian osteopaths who report sending referrals to podiatrists with those who do not report referring

\begin{tabular}{|c|c|c|c|c|}
\hline & Yes $(n=651)$ & No $(n=341)$ & $p$-value & Odds ratio $[\mathrm{OR}]^{*}$ \\
\hline \multicolumn{5}{|l|}{ Gender } \\
\hline Male & $393(60.4 \%)$ & $183(53.7 \%)$ & & \\
\hline Female & $258(39.6 \%)$ & $158(46.3 \%)$ & 0.04 & $0.76[0.59,0.99]$ \\
\hline \multicolumn{5}{|l|}{ Age (years) } \\
\hline Mean $( \pm S D)$ & $37.4( \pm 10.1)$ & $39.2( \pm 12.0)$ & $0.01^{\mathrm{a}}$ & \\
\hline \multicolumn{5}{|l|}{ Years in clinical practice } \\
\hline Mean $( \pm S D)$ & $11.4( \pm 8.3)$ & $11.4( \pm 10.2)$ & 0.99 & \\
\hline \multicolumn{5}{|l|}{ Patient care hours per week } \\
\hline Mean $( \pm S D)$ & $28.3( \pm 12.1)$ & $27.3( \pm 12.2)$ & 0.25 & \\
\hline \multicolumn{5}{|l|}{ Patient visits per week } \\
\hline Mean $( \pm S D)$ & $37.3( \pm 18.9)$ & $35.0( \pm 18.0)$ & 0.10 & \\
\hline \multicolumn{5}{|l|}{ Qualification (n, \%)^ } \\
\hline Diploma & $36(5.5 \%)$ & $26(7.6 \%)$ & 0.34 & \\
\hline Advanced diploma & $5(0.8 \%)$ & $4(1.2 \%)$ & & \\
\hline Bachelor degree & $144(22.1 \%)$ & $74(21.7 \%)$ & & \\
\hline Master's degree & $450(69.1 \%)$ & $231(67.7 \%)$ & & \\
\hline $\mathrm{PhD}$ & $2(0.3 \%)$ & $3(0.9 \%)$ & & \\
\hline Other & $14(2.2 \%)$ & $3(0.9 \%)$ & & \\
\hline \multicolumn{5}{|l|}{ Involved in as an osteopath } \\
\hline University teaching ${ }^{\#}$ & $73(11.2 \%)$ & $43(12.6 \%)$ & 0.52 & \\
\hline Clinical supervision" & $104(16.0 \%)$ & $46(13.5 \%)$ & 0.30 & \\
\hline Professional organisations & $65(10.0 \%)$ & $42(12.3 \%)$ & 0.26 & \\
\hline Research & $35(5.4 \%)$ & $19(5.6 \%)$ & 0.89 & \\
\hline Volunteer & $110(16.9 \%)$ & 49 (1.4\%) & 0.30 & \\
\hline
\end{tabular}

* unadjusted odds ratio, $\wedge$ analysed as a categorical variable, ${ }^{\#}$ participation in the previous 12 months (yes/no), ${ }^{a} \mathrm{~d}=0.17$ [0.04-0.30] 
Table 2 Comparison of practice characteristics of Australian osteopaths who report sending referrals to podiatrists with those who do not report referring

\begin{tabular}{|c|c|c|c|c|}
\hline & Yes $(n=651)$ & No $(n=341)$ & $p$-value & Odds Ratio $(\mathrm{OR})^{\mathrm{a}}[95 \% \mathrm{Cl}]$ \\
\hline \multicolumn{5}{|l|}{ Practice location } \\
\hline Urban practice & $538(82.6 \%)$ & $282(82.7 \%)$ & 0.98 & - \\
\hline More than one practice location & $216(33.2 \%)$ & $131(38.4 \%)$ & 0.10 & - \\
\hline \multicolumn{5}{|c|}{ Co-located with other health professionals ('yes') } \\
\hline Osteopath & $432(66.4 \%)$ & $211(61.9 \%)$ & 0.16 & - \\
\hline General Practitioner & $45(6.9 \%)$ & $27(7.9 \%)$ & 0.56 & - \\
\hline Specialist Medical Practitioner & $15(2.3 \%)$ & $16(4.7 \%)$ & 0.04 & $0.48[0.23,0.98]$ \\
\hline Podiatrist & $123(18.9 \%)$ & $24(7.0 \%)$ & $<0.01$ & $3.08[1.94,4.87]$ \\
\hline Physiotherapist & $100(15.4 \%)$ & $44(12.9 \%)$ & 0.30 & - \\
\hline Exercise Physiologist & $99(15.2 \%)$ & $25(7.3 \%)$ & $<0.01$ & $2.27[1.43,3.59]$ \\
\hline Occupational Therapist & $13(2.0 \%)$ & $6(1.8 \%)$ & 0.79 & - \\
\hline Psychologist & $120(18.4 \%)$ & $71(20.8 \%)$ & 0.36 & - \\
\hline Massage Therapist & $343(52.7 \%)$ & $158(46.3 \%)$ & 0.06 & - \\
\hline Acupuncturist & $116(17.8 \%)$ & $72(21.1 \%)$ & 0.21 & - \\
\hline Naturopath & $130(20.0 \%)$ & $63(18.5 \%)$ & 0.57 & - \\
\hline Dietician & $49(7.5 \%)$ & $23(6.7 \%)$ & 0.65 & - \\
\hline Nutritionist & $50(7.7 \%)$ & $28(8.2 \%)$ & 0.77 & - \\
\hline \multicolumn{5}{|c|}{ Send referrals to other health professionals ('yes') } \\
\hline Osteopath & $356(54.7 \%)$ & $150(44.0 \%)$ & $<0.01$ & $1.54[1.18,2.00]$ \\
\hline General Practitioner & $613(94.2 \%)$ & $265(77.7 \%)$ & $<0.01$ & $4.62[3.05,7.01]$ \\
\hline Specialist Medical Practitioner & $343(52.7 \%)$ & $100(29.3 \%)$ & $<0.01$ & $2.68[2.03,3.55]$ \\
\hline Podiatrist & - & - & - & - \\
\hline Physiotherapist & $266(40.9 \%)$ & $65(19.1 \%)$ & $<0.01$ & $2.93[2.15,4.00]$ \\
\hline Exercise Physiologist & $306(47.0 \%)$ & $92(27.0 \%)$ & $<0.01$ & $2.40[1.80,3.19]$ \\
\hline Occupational Therapist & $87(13.4 \%)$ & $19(5.6 \%)$ & $<0.01$ & $2.61[1.56,4.37]$ \\
\hline Psychologist & $265(40.7 \%)$ & $84(24.6 \%)$ & $<0.01$ & $2.10[1.57,2.81]$ \\
\hline Massage Therapist & $492(75.6 \%)$ & $179(52.5 \%)$ & $<0.01$ & $2.80[2.12,3.70]$ \\
\hline Acupuncturist & $335(51.5 \%)$ & $116(34.0 \%)$ & $<0.01$ & $2.05[1.56,2.70]$ \\
\hline Naturopath & $356(54.7 \%)$ & $121(35.5 \%)$ & $<0.01$ & $2.19[1.67,2.87]$ \\
\hline Dietician & $136(20.9 \%)$ & $31(9.1 \%)$ & $<0.01$ & $2.64[1.74,3.99]$ \\
\hline Nutritionist & $94(14.4 \%)$ & $35(10.3 \%)$ & 0.06 & - \\
\hline \multicolumn{5}{|c|}{ Receive referrals from other health professionals ('yes') } \\
\hline Osteopath & $429(65.9 \%)$ & $185(54.3 \%)$ & $<0.01$ & $1.63[1.24,2.13]$ \\
\hline General Practitioner & $594(91.2 \%)$ & $292(85.6 \%)$ & $<0.01$ & $1.75[1.16,2.62]$ \\
\hline Specialist Medical Practitioner & $168(25.8 \%)$ & $69(20.2 \%)$ & 0.05 & - \\
\hline Podiatrist & $418(64.2 \%)$ & $53(15.5 \%)$ & $<0.01$ & $9.75[6.98,13.61]$ \\
\hline Physiotherapist & $190(29.2 \%)$ & $76(22.3 \%)$ & 0.02 & $1.44[1.06,1.95]$ \\
\hline Exercise Physiologist & $196(30.1 \%)$ & $62(18.2 \%)$ & $<0.01$ & $1.94[1.40,2.67]$ \\
\hline Occupational Therapist & $47(7.2 \%)$ & $14(4.1 \%)$ & 0.05 & - \\
\hline Psychologist & $96(14.7 \%)$ & $58(17.0 \%)$ & 0.35 & - \\
\hline Massage Therapist & $529(81.3 \%)$ & $225(66.0 \%)$ & $<0.01$ & $2.23[1.66,3.01]$ \\
\hline Acupuncturist & $265(40.7 \%)$ & $105(30.8 \%)$ & $<0.01$ & $1.54[1.17,2.04]$ \\
\hline Naturopath & $285(43.8 \%)$ & $115(33.7 \%)$ & $<0.01$ & $1.53[1.16,2.01]$ \\
\hline Dietician & $28(4.3 \%)$ & $11(3.2 \%)$ & 0.41 & - \\
\hline Nutritionist & $37(5.7 \%)$ & $18(5.3 \%)$ & 0.79 & - \\
\hline \multicolumn{5}{|l|}{ Diagnostic imaging } \\
\hline Referral for imaging ('often') & $48(7.4 \%)$ & $25(7.3 \%)$ & 0.98 & - \\
\hline Investigation of unknown pathologies & $496(76.2 \%)$ & $246(72.1 \%)$ & 0.16 & - \\
\hline Investigation of suspected diagnosis & $562(86.3 \%)$ & $273(80.1 \%)$ & 0.01 & $1.57[1.11,2.22]$ \\
\hline
\end{tabular}


Table 2 (continued)

\begin{tabular}{|c|c|c|c|c|}
\hline & Yes $(n=651)$ & No $(n=341)$ & $p$-value & Odds Ratio $(\mathrm{OR})^{\mathrm{a}}[95 \% \mathrm{Cl}]$ \\
\hline Investigation of potential fractures & $496(76.2 \%)$ & $254(74.5 \%)$ & 0.55 & - \\
\hline Rule out risk factors prior to treatment & $174(26.7 \%)$ & $98(28.7 \%)$ & 0.50 & - \\
\hline General screening of the spine & $13(2.0 \%)$ & $19(5.6 \%)$ & $<0.01$ & $0.34[0.17,0.71]$ \\
\hline \multicolumn{5}{|l|}{ Patient assessment ('yes') } \\
\hline Orthopaedic testing & $646(99.2 \%)$ & $322(94.4 \%)$ & $<0.01$ & $7.62[2.82,20.60]$ \\
\hline Clinical assessment algorithm & $320(49.2 \%)$ & $148(43.4 \%)$ & 0.08 & - \\
\hline Neurological testing & $611(93.9 \%)$ & $307(90.0 \%)$ & 0.03 & $1.69[1.05,2.72]$ \\
\hline Screening questionnaire & $433(66.5 \%)$ & $200(58.7 \%)$ & 0.01 & $1.40[1.07,1.83]$ \\
\hline Cranial nerve testing & $443(68.0 \%)$ & $229(6.72 \%)$ & 0.77 & - \\
\hline \multicolumn{5}{|l|}{ Payment strategies } \\
\hline HICAPS & $603(92.8 \%)$ & $301(88.8 \%)$ & 0.03 & $1.62[1.03,2.54]$ \\
\hline Medicare EasyClaim & $302(46.4 \%)$ & $129(37.8 \%)$ & 0.01 & $1.42[1.09,1.86]$ \\
\hline
\end{tabular}

${ }^{\text {a }}$ unadjusted odds ratio

reported sending patient referrals to podiatrists $(O R 7.62$, 95\%CI 8.82-20.60) and these osteopaths were $40 \%$ more likely to use the Medicare EasyClaim system to claim rebates under the CDM scheme [13] (Table 2).

Australian osteopaths who reported sending referrals to podiatrists were more likely to report often treating low back pain (OR 3.11, 95\%CI 1.00-9.57), postural disorders (OR 1.71, 95\%CI 1.03-2.26) and tendinopathies (OR 1.38, 95\%CI 1.06-1.81), than their counterparts who did not send patient referrals to a podiatrist (Table 3 ).

\section{Discussion}

The current study identified the demographic, practice and clinical management characteristics of Australian osteopaths who report sending referrals to podiatrists, compared to their colleagues who do not report referring. A significant observation was the number of Australian osteopaths reporting sending referrals to podiatrists - approximately two-thirds reported sending referrals to podiatrists [5]. This observation, alongside the large association with receiving referrals from a podiatrist, suggests that a strong referral relationship exists between Australian osteopaths and osteopaths are utilising the expertise of podiatrists for patient care. These observations also potentially reflect a shared understanding between osteopaths and podiatrists of the role that each profession plays in patient care. Data from some studies indicate instances of multidisciplinary care involving podiatrists as part of the team [24-26]. These studies describe podiatrists positively contributing to the multidisciplinary care team for arthritic and ulcerative conditions; however, there is little literature describing multidisciplinary care involving osteopaths [27] and none in Australia.
Through secondary analysis of data from a national osteopathy PBRN, it appears that Australian osteopaths who report sending referrals to podiatrists are likely to engage in referrals with other allied health and complementary medicine professions. This finding is encouraging given the need for interprofessional patient care of musculoskeletal complaints [28], and has rarely been demonstrated in the literature describing the Australian osteopathy profession. The data presented here may be useful for informing health policy development around multidisciplinary care for musculoskeletal complaints (i.e. how best to utilise available health resources for patient care), and in patient care for chronic conditions given their significant cost to the healthcare system [29]. There is also an opportunity to use the data in the current study to inform the pre-registration education of Australian osteopaths and podiatrists, particularly focusing on interprofessional care.

Regarding practitioner characteristics, the current study identified two significant variables. The first was that female osteopaths were approximately $30 \%$ less likely to send referrals to podiatrists compared to their male counterparts in the unadjusted modelling. The reason for this difference in referrals based on gender requires additional exploration. Secondly, younger osteopaths were also more likely to report sending referrals to podiatrists compared to older colleagues. Whether this reflects an increase in knowledge of the role of podiatrists in musculoskeletal complaint care through pre-professional education, or experience from practice (or both) would need additional research. However, the finding is encouraging from the multidisciplinary care perspective.

Australian osteopaths who reported sending referrals to podiatrists were over seven times more likely 
Table 3 Comparison of clinical management characteristics of Australian osteopaths who report sending referrals to podiatrists with those who do not report referring

\begin{tabular}{|c|c|c|c|c|}
\hline & Yes $(n=651)$ & No $(n=341)$ & $p$-value & Odds Ratio $(\mathrm{OR}) *[95 \% \mathrm{Cl}]$ \\
\hline \multicolumn{5}{|l|}{ Discuss with patients ('often') } \\
\hline Diet & $239(36.8 \%)$ & $136(39.9 \%)$ & 0.34 & - \\
\hline Smoking and drug use & $115(1.7 \%)$ & $64(18.8 \%)$ & 0.68 & - \\
\hline Physical activity & $591(90.9 \%)$ & $295(86.5 \%)$ & 0.03 & $1.56[1.04,2.35]$ \\
\hline Occupation Health \& Safety & $346(53.4 \%)$ & $160(46.9 \%)$ & 0.05 & - \\
\hline Pain counselling & $175(26.9 \%)$ & $91(26.7 \%)$ & 0.95 & - \\
\hline Stress & $314(48.4 \%)$ & $175(51.5 \%)$ & 0.35 & - \\
\hline Nutrition & $158(24.3 \%)$ & $94(27.6 \%)$ & 0.26 & - \\
\hline Medication & $262(40.3 \%)$ & $129(37.9 \%)$ & 0.47 & - \\
\hline \multicolumn{5}{|l|}{ Patient presentations ('often') } \\
\hline Neck pain & $642(98.8 \%)$ & $329(96.5 \%)$ & 0.01 & $2.93[1.19,7.23]$ \\
\hline Thoracic pain & $600(92.3 \%)$ & $309(90.6 \%)$ & 0.36 & - \\
\hline Low back pain & $645(99.2 \%)$ & $332(97.6 \%)$ & 0.04 & $3.11[1.00,9.57]$ \\
\hline Hip musculoskeletal pain & $500(76.9 \%)$ & $244(71.8 \%)$ & 0.08 & - \\
\hline Knee musculoskeletal pain & $324(50.0 \%)$ & $167(49.1 \%)$ & 0.79 & - \\
\hline Ankle musculoskeletal pain & $226(34.9 \%)$ & $107(31.4 \%)$ & 0.27 & - \\
\hline Foot musculoskeletal pain & $197(30.4 \%)$ & $97(28.4 \%)$ & 0.53 & - \\
\hline Shoulder musculoskeletal pain & $539(83.1 \%)$ & $262(77.1 \%)$ & 0.02 & $1.46[1.05,2.02]$ \\
\hline Elbow musculoskeletal pain & $164(25.4 \%)$ & $87(25.6 \%)$ & 0.94 & - \\
\hline Wrist musculoskeletal pain & $122(18.8 \%)$ & $66(19.4 \%)$ & 0.84 & - \\
\hline Hand musculoskeletal pain & $74(11.5 \%)$ & $47(13.9 \%)$ & 0.27 & - \\
\hline Postural disorders & $469(72.4 \%)$ & $206(60.4 \%)$ & $<0.01$ & $1.71[1.03,2.26]$ \\
\hline Degenerative spine conditions & $411(63.4 \%)$ & $188(55.1 \%)$ & 0.01 & $1.41[1.08,1.84]$ \\
\hline Headache disorders & $673(68.0 \%)$ & $219(22.1 \%)$ & 0.43 & - \\
\hline Migraine disorders & $264(40.7 \%)$ & $136(40.0 \%)$ & 0.82 & - \\
\hline Spine health maintenance & $303(46.8 \%)$ & $155(45.5 \%)$ & 0.68 & - \\
\hline Chronic or persistent pain & $405(62.5 \%)$ & $225(66.0 \%)$ & 0.28 & - \\
\hline Tendinopathies & $286(44.1 \%)$ & $124(36.4 \%)$ & 0.02 & $1.38[1.06,1.81]$ \\
\hline Temporomandibular joint disorders & $115(17.7 \%)$ & $68(20.1 \%)$ & 0.37 & - \\
\hline Non-musculoskeletal disorders & $65(10.1 \%)$ & $61(18.0 \%)$ & $<0.01$ & $0.51[0.35,0.75]$ \\
\hline \multicolumn{5}{|l|}{ Patient subgroups (treat 'often') } \\
\hline Up to 3 years of age & $96(14.8 \%)$ & $60(17.6 \%)$ & 0.24 & - \\
\hline 4 to 18 years of age & $186(28.6 \%)$ & $84(24.6 \%)$ & 0.18 & - \\
\hline Over 65 years of age & $386(59.4 \%)$ & $186(54.5 \%)$ & 0.14 & - \\
\hline Aboriginal \& Torres Strait Islander peoples & $7(1.1 \%)$ & $0(0 \%)$ & 0.05 & - \\
\hline Pregnancy & $237(36.5 \%)$ & $107(31.4 \%)$ & 0.11 & - \\
\hline Non-English speaking & $24(3.7 \%)$ & $9(2.6 \%)$ & 0.38 & - \\
\hline Sport injuries & $350(53.8 \%)$ & $151(44.4 \%)$ & $<0.01$ & $1.46[1.12,1.90]$ \\
\hline Worker injury (compensable) & $68(10.5 \%)$ & $35(10.3 \%)$ & 0.91 & - \\
\hline Work injury (non-compensable) & $238(36.6 \%)$ & $103(30.2 \%)$ & 0.04 & $1.33[1.01,1.77]$ \\
\hline Traffic injury (compensable) & $36(5.6 \%)$ & $18(5.3 \%)$ & 0.85 & - \\
\hline Traffic injury (non-compensable) & $73(11.2 \%)$ & $41(12.1 \%)$ & 0.68 & - \\
\hline Post-surgery & $52(8.0 \%)$ & $27(8.0 \%)$ & 0.98 & - \\
\hline \multicolumn{5}{|l|}{ Manual therapy (use 'often') } \\
\hline Counterstrain & $293(45.1 \%)$ & $127(37.2 \%)$ & 0.01 & $1.38[1.06,1.81]$ \\
\hline Muscle energy technique & $546(84.0 \%)$ & $242(71.0 \%)$ & $<0.01$ & $2.15[1.57,2.94]$ \\
\hline High-velocity, low-amplitude manipulation & $441(67.8 \%)$ & $191(56.0 \%)$ & $<0.01$ & $1.65[1.26,2.17]$ \\
\hline Joint manipulation & $281(43.4 \%)$ & $112(32.8 \%)$ & $<0.01$ & $1.56[1.19,2.06]$ \\
\hline Soft tissue technique & $584(90.0 \%)$ & $264(77.4 \%)$ & $<0.01$ & $2.62[1.83,3.76]$ \\
\hline
\end{tabular}


Table 3 (continued)

\begin{tabular}{|c|c|c|c|c|}
\hline & Yes $(n=651)$ & No $(n=341)$ & $p$-value & Odds Ratio (OR)* $[95 \% \mathrm{Cl}]$ \\
\hline Myofascial release & $426(65.6 \%)$ & $186(54.5 \%)$ & $<0.01$ & $1.59[1.22,2.09]$ \\
\hline Visceral techniques & $56(8.6 \%)$ & $42(12.3 \%)$ & 0.06 & - \\
\hline Lymphatic pump & $52(8.0 \%)$ & $32(9.4 \%)$ & 0.46 & - \\
\hline Autonomic balancing & $93(14.3 \%)$ & $64(18.8 \%)$ & 0.06 & - \\
\hline Biodynamics & $88(13.5 \%)$ & $67(19.6 \%)$ & 0.01 & - \\
\hline Functional technique & $176(27.1 \%)$ & $94(27.6 \%)$ & 0.87 & - \\
\hline Balanced ligamentous tension & $216(33.2 \%)$ & $133(39.0 \%)$ & 0.07 & - \\
\hline Chapman's reflexes & $16(2.5 \%)$ & $8(2.4 \%)$ & 0.91 & - \\
\hline Trigger point therapy & $199(30.6 \%)$ & $59(17.4 \%)$ & $<0.01$ & - \\
\hline Osteopathy in the Cranial Field & $141(21.7 \%)$ & $92(27.0 \%)$ & 0.06 & - \\
\hline Facilitated positional release & $115(17.7 \%)$ & $51(15.0 \%)$ & 0.29 & - \\
\hline Dry needling & $170(26.2 \%)$ & $64(18.8 \%)$ & 0.01 & $1.52[1.10,2.11]$ \\
\hline Exercise prescription & $500(77.0 \%)$ & $233(68.3 \%)$ & $<0.01$ & $1.55[1.16,2.08]$ \\
\hline Shockwave therapy & $11(1.7 \%)$ & $7(2.1 \%)$ & 0.68 & - \\
\hline Ultrasound & $14(2.2 \%)$ & $13(3.8 \%)$ & 0.13 & - \\
\hline Transcutaneous Electrical Nerve Stimulation (TENS) & $7(1.1 \%)$ & $12(3.5 \%)$ & $<0.01$ & $0.29[0.11,0.76]$ \\
\hline Instrument manipulation & $0(0 \%)$ & $2(0.6 \%)$ & 0.05 & - \\
\hline Instrument soft tissue & $8(1.2 \%)$ & $4(1.2 \%)$ & 0.94 & - \\
\hline Sport taping & $93(14.3 \%)$ & $29(8.5 \%)$ & $<0.01$ & $1.79[1.15,2.79]$ \\
\hline \multicolumn{5}{|l|}{ Expanded practice scope ('definitely') } \\
\hline Prescribing rights & $175(26.9 \%)$ & $82(24.0 \%)$ & 0.32 & - \\
\hline Referral rights to orthopaedic surgeon & $475(73.1 \%)$ & $228(66.9 \%)$ & 0.04 & $1.34[1.01,1.79]$ \\
\hline Referral rights to paediatrician & $350(53.8 \%)$ & $190(55.7 \%)$ & 0.57 & - \\
\hline Referral rights to sports medicine specialist & $542(83.5 \%)$ & $248(72.7 \%)$ & $<0.01$ & $1.90[1.39,2.60]$ \\
\hline Referral rights to rheumatologist & $424(65.2 \%)$ & $205(60.1 \%)$ & 0.11 & - \\
\hline Referral rights to other medical specialist & $1(0.2 \%)$ & $0(0 \%)$ & 0.46 & - \\
\hline Expanded diagnostic imaging rights & $551(84.8 \%)$ & $271(79.5 \%)$ & 0.03 & $1.44[1.02,2.02]$ \\
\hline \multicolumn{5}{|l|}{ Research ('strongly agree') } \\
\hline Help patients understand osteopathy & $284(43.6 \%)$ & $159(46.6 \%)$ & 0.36 & - \\
\hline $\begin{array}{l}\text { Help general practitioners and other health professionals } \\
\text { understand osteopathy }\end{array}$ & $441(70.3 \%)$ & $229(70.5 \%)$ & 0.96 & - \\
\hline Provide scientific evidence & $324(52.4 \%)$ & $191(59.5 \%)$ & 0.04 & $0.75[0.57,0.98]$ \\
\hline Irrelevant to the development of osteopathy ${ }^{\mathrm{a}}$ & $348(56.4 \%)$ & $215(67.2 \%)$ & $<0.01$ & $0.63[0.47,0.84]$ \\
\hline
\end{tabular}

* unadjusted odds ratio, ' 'strongly disagree'

to utilise orthopaedic testing in patient assessment, compared to osteopaths who did not report referring. Orthopaedic testing is based on stressing musculoskeletal tissues and may be utilised to assist in developing a working diagnosis for the patient's complaint. Further, these tests are utilised by both podiatrists [30] and osteopaths [31]. It may be that this shared understanding of the orthopaedic tests relevant to the respective professions practice may be being captured in the current secondary analysis. Again, such an assertion would benefit from additional research to explore this potential shared understanding of musculoskeletal examination procedures.

From a clinical management perspective, Australian osteopaths who send referrals to podiatrists were over $70 \%$ more likely to report treating postural disorders. Studies have described the relationship between the prescription of foot orthoses and changes in posture in both healthy $[32,33]$ and clinical populations [34]. Although a direct relationship cannot be established from the current dataset, it may be that postural disorders are a commonly referred issue for shared management of patients given they are reported to be managed by over one-third of Australian osteopaths [7]. Whether postural disorders are a key driver of referrals would be an interesting avenue for further research. It would be valuable to understand the clinical reasoning of osteopaths and podiatrists in the management of postural disorders. Additional research could also be directed towards understanding 
other conditions that may result in frequent referrals between the two professions.

Australian osteopaths who reported sending referrals to podiatrists were less likely, than their non-referring colleagues, to report treating non-musculoskeletal conditions. The dominant practice of Australian osteopaths relates to the management of musculoskeletal complaints $[5,6]$. However, there is a subsection of the Australian osteopathic profession that apply manual therapy techniques to assist in the management of non-musculoskeletal complaints [5]. There is varying evidence to support the effectiveness of osteopathy care for non-musculoskeletal complaints [35, 36]. It may be that the nature of the non-musculoskeletal complaints managed by some Australian osteopaths does not require referral to a podiatrist. Rather, osteopaths appear to be referring to podiatrists for musculoskeletal complaints. The nature of the conditions resulting in referral to a podiatrist requires further investigation.

The bivariate analysis undertaken indicates the payment processes that might be associated with osteopathy and podiatry co-management of patients. Australian osteopaths who send referrals to podiatrists were over $40 \%$ more likely to use the Medicare EasyClaim service than osteopaths who do not send referrals. Both professions are eligible to treat patients under the CDM scheme, whereby patients with chronic diseases can access government funded care for up to five consultations with allied health professionals [13]. Medicare EasyClaim allows patients to claim the rebate at the time of the consultation upon referral from the patients' general practitioner. It may be that patients under this CDM scheme are utilising both osteopathy and podiatry services, and the increased use of Medicare EasyClaim reflects this. This assertion may be supported by the large association observed in the current secondary analysis with osteopaths sending referrals to general practitioners. Additional investigations would assist in testing these assertions to understand how osteopathy and podiatry services are used under the CDM scheme.

The limitation of the current research is associated with the cross-sectional nature of the questionnaire. Questionnaires used with these study designs are potentially susceptible to social desirability and acquiescence biases [37], and the latter may have influenced responses to the send and receive referral items on the questionnaire. Further, it is not possible to comment on the frequency of referrals between the two professions given the dichotomous nature of the responses to these items.

\section{Conclusion}

This study offers an initial empirical examination of the referral relationships between Australian osteopaths and podiatrists. Our secondary analysis shows Australian osteopaths who report sending referrals to podiatrists are also more likely to engage in referrals with other allied health and complementary medicine professions. The data presented here offers a resource for informing health policy development and has the potential for use in the pre-registration education of Australian osteopaths and podiatrists. Further research could develop a deeper understanding of the nature and frequency of the referrals between Australian osteopaths and podiatrists, including how these two professions work together through the Medicare CDM scheme for the benefit of patients.

\section{Acknowledgements}

The authors would like to acknowledge the support of Distinguished Professor Jon Adams, Dr. Amie Steel and Dr. Wenbo Peng as part of the ORION PBRN Scientific Committee.

\section{Authors' contributions}

MF and BV devised the study. MF and BV undertook the data analysis. All authors developed the literature review and discussion. All authors approved the final version of the manuscript.

\section{Funding}

Open Access funding enabled and organized by CAUL and its Member Institutions The ORION project is funded by Osteopathy Australia. The funding source had no influence in the design of the study and collection, analysis, and interpretation of data and in writing the manuscript. The research reported in this paper is the sole responsibility of the authors and reflects the independent ideas and scholarship of the authors alone.

\section{Availability of data and materials}

The datasets analysed during the current study are not publicly available as the authors do not have the authority to disseminate the data. The dataset is available through reasonable request at arccim@uts.edu.au.

\section{Declarations}

Ethics approval and consent to participate

Ethics approval for the current study was provided by the University of Technology Sydney (\#2014000759). The University of Technology Sydney Human Research Ethics Committee is registered with the Australian Government National Health and Medical Research Council (EC00146, https://www.nhmrc. gov.au/research-policy/ethics/human-research-ethics-committees). The research was performed in accordance with the Declaration of Helsinki. All participants in the study provided informed consent.

\section{Consent for publication}

Not applicable.

\section{Competing interests}

The authors report no competing interests in relation to the manuscript.

\section{Author details \\ ${ }^{1}$ College of Health and Biomedicine, Victoria University, Melbourne, Australia. ${ }^{2}$ School of Public Health, University of Technology Sydney, Sydney, Australia. ${ }^{3}$ Department of Medical Education, University of Melbourne, Melbourne, Australia. ${ }^{4}$ School of Health and Human Sciences, Southern Cross University, Lismore, Australia. ${ }^{5}$ School of Allied Health, Human Services and Sport, La Trobe University, Melbourne, Australia.}


Received: 22 December 2020 Accepted: 25 November 2021

Published online: 18 February 2022

\section{References}

1. NSW Health. Multidisciplinary Team Care 2014. https://www.health.nsw. gov.au/healthone/Pages/multidisciplinary-team-care.aspx. Accessed 13th May 2020.

2. Leach B, Morgan P, de Oliveira JS, Hull S, Østbye T, Everett C. Primary care multidisciplinary teams in practice: a qualitative study. BMC Family Pract. 2017;18:115.

3. Bonciani M, Barsanti S, Murante AM. Is the co-location of GPs in primary care centres associated with a higher patient satisfaction? Evidence from a population survey in Italy. BMC Health Serv Res. 2017:17:248.

4. Rumball-Smith J, Wodchis WP, Koné A, Kenealy T, Barnsley J, Ashton T. Under the same roof: co-location of practitioners within primary care is associated with specialized chronic care management. BMC Family Pract. 2014;15:149.

5. Adams J, Sibbritt D, Steel A, Peng W. A workforce survey of Australian osteopathy: analysis of a nationally-representative sample of osteopaths from the osteopathy research and innovation network (ORION) project. BMC Health Serv Res. 2018;18:352. https://doi.org/10.1186/ s12913-018-3158-y.

6. Burke SR, Myers R, Zhang AL. A profile of osteopathic practice in Australia 2010-2011: a cross sectional survey. BMC Musculo Disorder. 2013;14:227. https://doi.org/10.1186/1471-2474-14-227.

7. Orrock P. Profile of members of the Australian osteopathic association: part 2 - the patients. Int J Osteopath Med. 2009;12:128-39. https://doi. org/10.1016/j.josm.2009.06.001

8. Podiatry Board of Australia. Accreditation. 2019. https://www.podiatrybo ard.gov.au/Accreditation.aspx. Accessed 22 Apr 2020.

9. Osteopathy Board of Australia. Accreditation. 2016. https://www.osteo pathyboard.gov.au/Accreditation.aspx. Accessed 22 Apr 2020.

10. Podiatry Board of Australia. Registrant Data (Reporting period: 01 July 2019 to 30 September 2019). 2019. https://www.podiatryboard.gov.au/ About/Statistics.aspx. Accessed 13 Feb 2020.

11. Osteopathy Board of Australia. Registrant Data (Reporting period: 01 July 2019 to 30 September 2019). 2019. https://www.osteopathyboard.gov. au/About/Statistics.aspx. Accessed 13 Feb 2020.

12. Department of Health. Podiatry - Health workforce data. 2016. https:// hwd.health.gov.au/webapi/customer/documents/factsheets/2016/Podia try\%20-\%202016.pdf. Accessed 13 May 2020.

13. Department of Health. Chronic disease management (formerly enhanced primary care or EPC) — GP services. 2017. http://www.health.gov.au/ internet/main/publishing.nsf/Content/mbsprimarycare-chronicdiseasem anagement. Accessed 21 Dec 2017.

14. Menz HB. Utilisation of podiatry services in Australia under the Medicare enhanced primary care program, 2004-2008. J Foot Ankle Res. 2009;2:30.

15. Department of Health. Medicare item reports. 2020. http://medicarestatist ics.humanservices.gov.au/statistics/mbs_item.jsp. Accessed 16 Feb 2020.

16. Pirotta M, Temple-Smith M. Practice-based research networks. Aust Fam Physician. 2017:46:793-5.

17. Mold JW, Peterson KA. Primary care practice-based research networks: working at the interface between research and quality improvement. Ann Fam Med. 2005;3:S12-20.

18. Adams J, Lauche R, Peng W, Steel A, Moore C, Amorin-Woods L, et al. A workforce survey of Australian chiropractic: the profile and practice features of a nationally representative sample of 2,005 chiropractors. BMC Complement Altern Med. 2017;17:14. https://doi.org/10.1186/ s12906-016-1542-x.

19. Hickner J, Green LA. Practice-based research networks (PBRNs) in the United States: growing and still going after all these years. J Am Board Fam Med. 2015;28:541-5. https://doi.org/10.3122/jabfm.2015.05.150227.

20. Selby K, Cornuz J, Senn N. Establishment of a representative practicebased research network (PBRN) for the monitoring of primary care in Switzerland. J Am Board Fam Med. 2015;28:673-5. https://doi.org/10. 3122/jabfm.2015.05.150110.

21. Gilbert GH, Williams OD, Rindal DB, Pihlstrom DJ, Benjamin PL, Wallace MC, et al. The creation and development of the dental practice-based research network. J Am Dent Assoc. 2008;139:74-81.
22. Steel A, Peng W, Sibbritt D, Adams J. Introducing national osteopathy practice-based research networks in Australia and New Zealand: an overview to inform future osteopathic research. Sci Rep. 2020;10:846. https:// doi.org/10.1038/s41598-020-57918-7.

23. Paterson KL, Harrison C, Britt H, Hinman RS, Bennell KL. Management of foot/ankle osteoarthritis by Australian general practitioners: an analysis of national patient-encounter records. Osteoarthr Cartil. 2018;26:888-94.

24. Ndosi M, Ferguson R, Backhouse MR, Bearne L, Ainsworth P, Roach A, et al. National variation in the composition of rheumatology multidisciplinary teams: a cross-sectional study. Rheumatol Int. 2017;37:1453-9.

25. Cassidy S, Coda A, West K, Hendry G, Grech D, Jones J, et al. Confidence amongst multidisciplinary professionals in managing paediatric rheumatic disease in Australia. Arthritis. 2018;2018:1-4. https://doi.org/10. 1155/2F2018/2F7807490

26. Butters T. Interdisciplinary chronic-wound care services involving podiatry-a strengthened model of care? Wound Pract Res. 2011;19:229.

27. Morin C, Desrosiers J, Gaboury I. Enablers and barriers to the development of interprofessional collaboration between physicians and osteopaths: a mixed methods study. J Interprof Care. 2018;32:463-72. https:// doi.org/10.1080/13561820.2018.1435515.

28. Mior S, Barnsley J, Boon H, Ashbury FD, Haig R. Designing a framework for the delivery of collaborative musculoskeletal care involving chiropractors and physicians in community-based primary care. J Interprof Care. 2010;24:678-89. https://doi.org/10.3109/13561821003608757.

29. Australian Institute of Health and Welfare. Australia's Health 2020: in brief. 2020. https://www.aihw.gov.au/reports/australias-health/australiashealth-2020-in-brief/contents/summary. Accessed 20 Aug 2020.

30. Jarvis HL, Nester CJ, Jones RK, Williams A, Bowden PD. Inter-assessor reliability of practice based biomechanical assessment of the foot and ankle. J Foot Ankle Res. 2012;5:14. https://doi.org/10.1186/1757-1146-5-14.

31. Vaucher P, Macdonald RJD, Carnes D. The role of osteopathy in the Swiss primary health care system: a practice review. BMJ Open. 2018;8. https:// doi.org/10.1136/bmjopen-2018-023770.

32. Olmsted LC, Hertel J. Influence of foot type and orthotics on static and dynamic postural control. J Sport Rehabil. 2004;13:54-66.

33. Wilson ML, Rome K, Hodgson D, Ball P. Effect of textured foot orthotics on static and dynamic postural stability in middle-aged females. Gait Posture. 2008;27:36-42.

34. Simonsen MB, Oliveira ASC, Næsborg-Andersen K, Kowalski MR, HørslevPetersen K, Andersen MS. Patient-specific foot orthotics improves postural control of rheumatoid arthritis patients: a pilot study. Footwear Sci. 2017;9:S17-8.

35. Guillaud A, Darbois N, Monvoisin R, Pinsault N. Reliability of diagnosis and clinical efficacy of visceral osteopathy: a systematic review. BMC Complement Altern Med. 2018;18:65.

36. Müller A, Franke H, Resch K-L, Fryer G. Effectiveness of osteopathic manipulative therapy for managing symptoms of irritable bowel syndrome: a systematic review. J Osteopath Med. 2014;114:470-9. https://doi.org/10 7556/jaoa.2014.098.

37. Lavrakas P. Encyclopedia of survey research methods. California: Sage Publications; 2008

\section{Publisher's Note}

Springer Nature remains neutral with regard to jurisdictional claims in published maps and institutional affiliations. 